\title{
Optimal Design and Control
}

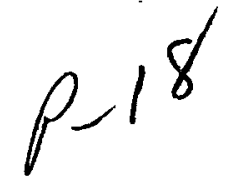

Proceedings of the Workshop on Optimal Design and Control

Blacksburg, Virginia

April 8-9, 1994

Jeffrey Borggaard, John Burkardt, Max Gunzburger, and Janet Peterson

Editors

(NASA-CR-199714) OPTIMAL CONTROL OF THERMALLY COUPLED NAVIER STOKES N96-15126 EQUATIONS (North Carolina State Univ.) $18 \mathrm{p}$

\author{
Unclas \\ $63 / 630079421$
}

Birkhäuser

Boston • Basel • Bèrlin 
Editors:

Jeffrey Borggaard

John Burkardt

Max Gunzburger

Janet Peterson

Air Force Center for Optimal Design and Control

Interdisciplinary Center for Applied Mathematics

Virginia Tech

Blacksburg, Virginia 24061-0531

Contributors

Preface .

Multilevel A

N. Alexâ

Shape Optirr

E. Arian

Library of Congress Cataloging In-Publication Data

Workshop on Optimal Design and Control (1994 : Blacksburg, VA.)

Optimal design and control : proceedings of the workshop on

Optimal Design and Control, Blacksburg, Virginia, April 8-9, 1994 /

Jeffrey Borggaard...[et al.], editors.

p. cm. - (Progress in systems and control theory ; v. 19)

Includes bibliographical references.

ISBN 0-8176-3808-3 (acid-free paper).

1. Automatic control--Congresses. 2. Mathematical optimization-

-Congresses. I. Borggaard, Jeffrey, 1964- . II. Title.

III. Series.

TJ212.W675 1994

629.8--dc20

$95-10230$

CIP

Printed on acid-free paper

(C) 1995 Birkhäuser Boston

Birkhäuser "Q⿻丅⿵冂⿰⿱丶㇀⿱㇒丶亅

Copyright is not claimed for works of U.S. Government employees.

All rights reserved. No part of this publication may be reproduced, stored in a retrieval system, or transmitted, in any form or by any means, electronic, mechanical, photocopying, recording, or otherwise, without prior permission of the copyright owner.

Permission to photocopy for internal or personal use of specific clients is granted by Birkhäuser Boston for libraries and other users registered with the Copyright Clearance Center (CCC), provided that the base fee of $\$ 6.00$ per copy, plus $\$ 0.20$ per page is paid directly to CCC, 222 Rosewood Drive, Danvers, MA 01923, U.S.A. Special requests should be addressed directly to Birkhäuser Boston, 675 Massachusetts Avenue, Cambridge, MA 02139, U.S.A.

ISBN 0-8176-3808-3

ISBN 3-7643-3808-3

Typeset by the editors

Printed and bound by Quinn-Woodbine, Woodbine, NJ

Printed in the U.S.A.

987654321

Computation

Nonlinear $\mathrm{Pr}$ J. Arora,

Optimal Con

J. Berg, I

The Applical

J. Betts

Algorithms f

J. Borgg:

H. Kim,

Generalized

Trajectory $\mathrm{O}$

K. Brena

The Optimal

M. Delfc

Uniformly S,

R. Fabiar

Implicit Filte P. Gilmo:

Generating $C$ A. Griew 


\title{
Optimal Control of Thermally Coupled Navier Stokes Equations
}

\author{
Kazufumi Ito, Jeffrey S. Scroggs, and Hien T. Tran \\ Center for Research in Scientific Computation \\ North Carolina State University \\ Raleigh NC 27695-8205, USA
}

bution by shape vity analysis for 2, 1992, 13-25. al contact probonal, J. Nonlin-

on between two to appear.

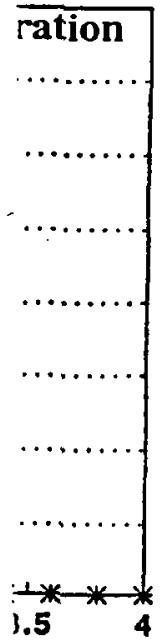

The optimal boundary temperature control of the stationary thermally coupled incompressible NavierStokes equation is considered. Well-posedness and existence of the optimal control and a necessary optimality condition are obtained. Optimization algorithms based on the augmented Lagrangian method with second order update are discussed. A test example motivated by control of transport process in the high pressure vapor transport (HPVT) reactor is presented to demonstrate the applicability of our theoretical results and proposed algorithm.

\section{Introduction}

In this paper we discuss the optimal control problem of the stationary thermally coupled incompressible Navier-Stokes equations. Consider the following optimal control problem

$$
\text { minimize } J(g)=\varphi\left(u, T-T_{0}\right)+\frac{\beta}{2}\left|g-T_{0}\right|_{L^{2}\left(\Gamma_{1}\right)}^{2}, \quad g \in \mathcal{C}
$$

subject to

$$
\begin{gathered}
u \cdot \nabla u+\nabla p=\nu \Delta u+\gamma\left(T-T_{0}\right) e_{d}+f, \\
\nabla \cdot u=0,\left.\quad u\right|_{\Gamma}=0, \\
u \cdot \nabla T=\nabla \cdot(\kappa \nabla T), \\
T=T_{0} \text { on } \Gamma_{0} \text { and } n \cdot \nabla T=H(g-T) \text { on } \Gamma_{1},
\end{gathered}
$$

where $f \in L^{2}(\Omega)^{d}$ is a source field, $u, p, T$ stand for the nondimensionalized velocity vector in $R^{d}$ with $d=2,3$, pressure, and temperature, respectively and $\mathcal{C}$ is the closed convex set in $L^{2}\left(\Gamma_{1}\right)$ such that

$$
\bar{T}_{1} \leq g \leq \bar{T}_{2} \text { on } \Gamma_{1}
$$

Here, $\bar{T}_{1} \leq T_{0} \leq \bar{T}_{2}$ and $\Gamma_{i}, i=0,1$ are disjoint open sets in $\Gamma$ such that $\Gamma=$ $\overline{\Gamma_{0}} \cup \overline{\Gamma_{l}}$. The constant $\gamma$ is given by $\gamma=\frac{\bar{g}}{T_{0}}$ where $T_{0}>0$ is a constant reference temperature and $\bar{g}$ is the gravitational constant, and $e_{d}$ denotes the $d$-th unit vector of $R^{d}$. Throughout this paper we assume that $\Omega$ is sufficiently smooth and $v, k$ and $H$ are positive constants.

This control problem is motivated by control of transport and growth processes in the high pressure vapor transport (HPVT) reactor [13]. For example, we may 
consider the Scholz geometry (Figure 1 in [13]). The source material and the growing crystal are sealed in a fused silica ampoule that is heated by a furnace liner at its outer cylindrical surface. The substrate $\Gamma_{0}$ (the single crystal) is located on a fused silica window (the bottom of the ampoule) which is cooled by a jet of helium gas from the outer surface. HPVT processes are based on physical vapor transport and can be described very roughly as proceeding via evaporation at the polycrystalline source and condensation at the surface of the cooler substrate. The system (1)-(2) is called the Boussinesq equations, where we assume the flow is incompressible and the transport phenomena of a single (carrier) gas is modeled. At the wall we assume Newton's law of cooling holds.

The cost-functional can be of tracking type

$$
\varphi\left(u, T-T_{0}\right)=\int_{\Omega}\left|u-u_{d}\right|^{2}+\left|T-T_{d}\right|^{2} d x
$$

where $\left(u_{d}, T_{d}\right)$ is the desired state, or minimization of friction force of flow in a subregion $\Omega_{1}$ of $\Omega$

$$
\varphi\left(u, T-T_{0}\right)=\int_{\Omega_{1}}|\nabla \times u|^{2} d x .
$$

\section{Well-posedness}

In this section, we discuss existence and uniqueness of solutions to (2). Let $U=$ $L^{2}\left(\Gamma_{1}\right)$ be the control space, $V=V_{0} \times V_{1}$ where $V_{0}$ is the divergence-free subspace of $\left(H_{0}^{1}(\Omega)\right)^{d}[7]$ and

$$
V_{1}=H_{\Gamma_{0}}^{1}=\left\{\phi \in H^{1}(\Omega):\left.\phi\right|_{\Gamma_{0}}=0\right\}
$$

and set $H=H_{0} \times L^{2}(\Omega) . H_{0}$ is the closure of $V_{0}$ with respect to the $L^{2}(\Omega)^{d}$-norm, and is defined by

$$
H_{0}=\left\{\phi \in L^{2}(\Omega)^{d}: \nabla \cdot \phi=0 \text { and } n \cdot \phi=0 \text { on } \Gamma\right\} .
$$

$H$ is equipped with the natural $L^{2}$-norm and $V$ is equipped with the norm

$$
|(\phi, \chi)|_{V}^{2}=|\phi|_{V_{0}}^{2}+|\chi|_{V_{1}}^{2}
$$

where

$$
|\phi|_{V_{0}}^{2}=|\nabla \phi|_{L^{2}(\Omega)}^{2} \text { and }|\chi|_{V_{1}}^{2}=|\nabla \chi|_{L^{2}(\Omega)}^{2}+H|\chi|_{L^{2}\left(\Gamma_{1}\right)}^{2}
$$

for $\psi=(\phi, \chi) \in V$. Define the trilinear form $b$ on $H^{1}(\Omega)^{d}$ by

$$
b\left(\phi_{1}, \phi_{2}, \phi_{3}\right)=\left\langle\phi_{1} \cdot \nabla \phi_{2}, \phi_{3}\right\rangle
$$

for $\phi_{i} \in H^{1}(\Omega)^{d}, i=1,2,3$. Then we have 
ind the growce liner at its ed on a fused if helium gas iransport and lycrystalline istem (1)-(2) incompress. At the wall

of flow in a

\section{2). Let $U=$} ee subspace

$(\Omega)^{d}$-norm,

crm
Lemma 1 The trilinear form is satisfies

(a) $\left|b\left(\phi_{1}, \dot{\phi}_{2}, \dot{\varphi}_{3}\right)\right| \leq\left|\phi_{1}\right|_{L^{+}} \mid \nabla\left(\left.\dot{\phi}_{2}\right|_{L^{2}}\left|\dot{\phi}_{3}\right|_{L^{+}} \leq M_{1}\left|\dot{\phi}_{1}\right|_{H^{1}}\left|\phi_{2}\right|_{H^{1}}\left|\dot{\phi}_{3}\right|_{H^{\prime}}\right.$

(b) $b\left(\phi_{1}, \dot{\phi}_{2}, \phi_{3}\right)+b\left(\phi_{1}, \phi_{3}, \dot{\phi}_{2}\right)=0$ provided that $\nabla \cdot \dot{\phi}_{1}=0$ ) and $\left(n \cdot \phi_{1}\right)\left(\dot{\phi}_{2} \cdot \dot{\phi}_{3}\right)=0$ on $\Gamma$

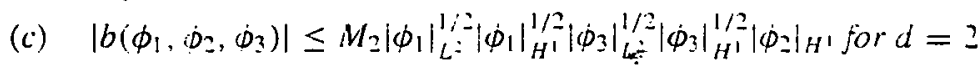

(d) $\left|b\left(\dot{\phi}_{1}, \phi_{2}, \dot{\phi}_{3}\right)\right| \leq M_{2}\left|\dot{\varphi}_{1}\right|_{L^{2}}^{1 / 4}\left|\phi_{1}\right|_{H^{1}}^{3 / 4}\left|\phi_{3}\right|_{L^{2}}^{3^{4}}\left|\phi_{3}\right|_{H^{1}}^{3 / 4} \mid \dot{\varphi}_{2} i_{H^{\prime}}$ for $d=3$

for $\dot{\phi}_{i} \in H^{\prime}(\Omega)^{d} . i=1,2,3$

Proof: By Green's tormula

$$
b\left(\dot{\phi}_{1}, \phi_{2}, \phi_{3}\right)+b\left(\dot{\phi}_{1}, \phi_{3}, \phi_{2}\right)=\left(\dot{\phi}_{1}, \nabla\left(\dot{\phi}_{2} \cdot \dot{\phi}_{3}\right)\right)=\left(n \cdot \phi_{1}, \dot{\phi}_{1} \cdot \dot{\phi}_{3}\right)_{\Gamma}
$$

for $\phi_{2} \in C^{1}(\Omega)$ and $\nabla \cdot \phi_{1}=0$. Hence (b) foilows from the continuity of $b$. The last two assertions follow from the fact that $|\psi|_{L^{+}} \leq\left. c\left|\varphi^{\prime}\right|_{L^{\prime}}^{1 / 2}|\psi| \psi\right|_{H^{\prime}} ^{1 / 2}$ tor $d=2$ and $|\psi|_{L^{+}} \leq c|\psi|_{L^{2}}^{3 / 4}|\psi|_{H^{1}}^{1 / 4}$ for $\psi \in H^{1}(\Omega)$ and some constant $c$.

In particular, Lemma 1 implies that

$$
b(u, \phi . \phi)=0 \text { for } u . \phi \in V_{0} \text {. }
$$

The weak or variational form of (2) is given by

$$
v(\nabla u, \nabla \phi)+b(u, u, \phi)=\left(f+\gamma\left(T-T_{0}\right) e_{a} . \phi\right)
$$

for all $\phi \in V_{0}$ and

$$
\begin{aligned}
\kappa\left(\nabla\left(T-T_{0}\right), \nabla \chi\right) & +\left\langle u \cdot \nabla\left(T-T_{0}\right), \chi\right\rangle v_{1} \times V_{1} \\
+ & \kappa\left(H\left(T-T_{0}\right), \chi\right)_{\Gamma_{1}}=\kappa\left(H\left(g-T_{0}\right), \chi\right)_{\Gamma_{1}} .
\end{aligned}
$$

for all $\chi \in V_{1}$. The pair $\left(u, T-T_{0}\right) \in V$ is said to be a weak solution of (2) if (6), (7) holds for all $\psi=(\phi, \chi) \in V$. Then we have the following theorem.

Theorem 2 Given $g \in L^{2}\left(\Gamma_{1}\right)$ there exists a weak solution $\left(u . T-T_{0}\right) \in V$ to (2) and

$$
\left|\left(u . T-T_{0}\right)\right|_{v} \leq \text { const }\left(|f|_{L^{2}}+|g|_{L^{2}\left(\Gamma_{1}\right)}\right) .
$$

Moreover, if $\bar{T}_{1} \leq g(x) \leq \bar{T}_{2}$ a.e. in $x \in \Gamma_{1}$ then $\bar{T}_{1} \leq T(x) \leq \bar{T}_{2}$ a.e in $\Omega$ for every solution $\left(u, T-T_{0}\right) \in V$.

Step I: (Existence) We show that (6), (7) has a solution $z=\left(u . T-T_{0}\right) \in V$. Given $\bar{u} \in V_{0}$, we consider the linear equation

$$
v(\nabla u, \nabla \phi)+\dot{b}(\bar{u}, u, \phi)=\left(\gamma\left(T-T_{0}\right) e_{d}+f, \phi\right) \text { for } \phi \subseteq V_{0}
$$




$$
\begin{aligned}
\kappa\left(\nabla\left(T-T_{0}\right), \nabla \chi\right)+\left\langle\vec{u} \cdot \nabla\left(T-T_{0}\right), \chi\right\rangle & +\kappa\left(H\left(T-T_{0}\right), \chi\right) \\
& =\kappa\left(H\left(g-T_{0}\right), \chi\right) r_{1},
\end{aligned}
$$

for $\chi \in V_{1}$. First, we show that (8),(9) has the unique solution $\left(u, T-T_{0}\right) \in$ $V$. Then, we show that the soiution map $S$ on $V_{0}$ defined by $S(\bar{u})=u$ where $\left(u, T-T_{0}\right) \in V$ is the unique solution to (8),(9) has a fixed point by Schauder fixed point theorem. The fixed point $u \in V_{0}$ and the corresponding solution $T-T_{0} \in V_{1}$ define a solution to (6), (7). By Green's formula

$$
\langle\bar{u} \cdot \nabla \theta, \chi\rangle+\langle\bar{u} \cdot \nabla \chi, \theta\rangle=0 \text { and in particular }\langle\bar{u} \cdot \chi, \chi\rangle=0
$$

for $\bar{u} \in V_{0}$, and $\theta, \chi \in H^{1}(\Omega)$. Hence, from Lemma 1 the sesquilinear form

$$
\kappa\left(\nabla \chi_{1}, \nabla \chi_{2}\right)+\left\langle\bar{u} \cdot \nabla \chi_{1}, \chi_{2}\right\rangle+\left(\kappa H \chi_{1}, \chi_{2}\right) \Gamma_{1}=0
$$

on $V_{1} \times V_{1}$ is bounded and $V_{1}$-coercive. It thus follows from the Lax-Milgram theorem that equation (9) has a unique solution $T-T_{0} \in V_{1}$. Choosing $\chi=T-T_{0}$ in (9), we have (independent of $\bar{u} \in V_{0}$ )

$$
\left|T-T_{0}\right|_{V_{1}}^{2} \leq H\left|g-T_{0}\right|_{L^{2}\left(\Gamma_{1}\right)}^{2} .
$$

Next, the sesquilinear form on $V_{0} \times V_{0}$ defined by

$$
\nu\left(\nabla \phi_{1}, \nabla \phi_{2}\right)+b\left(\bar{u}, \phi_{1}, \phi_{2}\right)
$$

is bounded and $V_{0}$-coercive from Lemma 1 and (5). Thus, by the Lax-Milgram theorem, equation (8) has a unique solution $u \in V_{0}$, and we have

$$
|u|_{v_{0}} \leq \frac{M_{3}}{v}\left(|f|_{L^{2}}+\gamma\left|T-T_{0}\right|_{L^{2}}\right)
$$

where $|\phi|_{H_{0}} \leq M_{3}|\phi|_{V_{0}}, \phi \in V_{0}$. Let $C$ be a closed convex subspace of $V_{0}$, defined by

$$
C=\left\{\phi \in V_{0}:|\phi|_{V_{0}} \leq \frac{M_{3}}{v}\left(|f|_{L^{2}}+\gamma M_{4} H\left|g-T_{0}\right|_{L^{2}\left(\Gamma_{1}\right)}\right\},\right.
$$

where $|\chi|_{L^{2}} \leq M_{4}|\chi|_{V_{1}}$ for $\chi \in V_{1}$. Then it follows from (11)-(12) that $S$ maps from $C$ into $C$. Moreover, the solution map $S$ is compact. In fact, if $\bar{u}_{k}$ converges weakly to $\vec{u}$ in $V_{0}$ then $\left|\bar{u}_{k}-\bar{u}\right|_{L^{4}} \rightarrow 0$ since $H^{l}(\Omega)$ is compactly embedded into $L^{4}(\Omega)$. Let $\left(u_{k}, T_{k}-T_{0}\right) \in V$ and $\left(u, T-T_{0}\right) \in V$ be the corresponding solution of (8),(9), respectively to $\bar{u}_{k} \in V_{0}$ and $\bar{u} \in V_{0}$. Then we have

$$
\kappa\left(T_{k}-T, \chi\right)_{V_{1}}+\left\langle\left(\bar{u}_{k}-\bar{u}\right) \cdot \nabla\left(T-T_{0}\right)+\bar{u}_{k} \cdot \nabla\left(T_{k}-T\right), \chi\right\rangle=0
$$

for $\chi \in V_{1}$ from Lemma 1 and (10)

$$
\kappa\left|T_{k}-T\right|_{V_{1}} \leq M_{1}\left|\bar{u}_{k}-\bar{u}\right|_{L^{4}}\left|T-T_{0}\right|_{V_{1}}
$$

Thermally $C_{1}$

which implies

and thus $\mid u_{k}-$ there exists at

Step 2: $\left(L^{\infty} \mathrm{e}\right.$

for all solutio: $\chi \in V_{1}[19]$ a:

Since from (1

where

Thus, we obta can prove tha

We have : $f$ and $g-T_{0}$.

Theorem 3 l unique solutic

Proof: Suppo have

$v(\nabla \tilde{u}$, $\kappa(\nabla \tilde{T}$

for $\phi \in V_{0}$ an $\phi=\tilde{u}$ and $\chi$

If we set $X=$

$$
(v-M \text { : }
$$


)$, x)$

). $x)_{\Gamma_{1}}, \quad(9)$

!, $\left.T-T_{0}\right) \in$ $1=u$ where chauder fixed ı $T-T_{0} \in V_{1}$

$=0$

ear form

Lax-Milgram $\mathrm{g} \chi=T-T_{0}$

-ax-Milgram

space of $V_{0}$,

1,

, that $S$ maps $\bar{u}_{k}$ converges nbedded into ding solution

c) $=0$ which implies $\left|T_{k}-T\right|_{v_{1}} \rightarrow 0$. Similarly, we have

$$
\nu\left|u_{k}-u\right|_{v_{0}} \leq M_{1}\left|\bar{u}_{k}-\bar{u}\right|_{L^{*}}|u|_{v_{0}}+\gamma M_{3}\left|T_{k}-T\right|_{L^{2}}
$$

and thus $\left|u_{k}-u\right|_{v_{0}} \rightarrow 0$. Now, by the Schauder fixed point theorem (e.g, see [19]) there exists at least one solution to (6), (7).

Step 2: ( $L^{\infty}$ estimate) We show that if $\bar{T}_{1} \leq g \leq \overline{\mathcal{F}}_{2}$ then

$$
\bar{T}_{1} \leq T \leq \bar{T}_{2} \text { a.e. } x \stackrel{x}{\in} \Omega \text {. }
$$

for all solutions $\left(u, T-T_{0}\right) \in V$ to (6), (7). In fact, let $\chi=\inf \left(T, \bar{T}_{1}\right)$. Then $\chi \in V_{1}[19]$ and we have from (6), (7)

$$
\kappa(\nabla T, \nabla \chi)+\langle u \cdot \nabla T, \chi\rangle+(\kappa H(T-g), \chi)_{\Gamma_{1}}=0 .
$$

Since from (10) $\langle u \cdot \nabla T, x\rangle=0$ we have

$$
\kappa(\nabla \chi, \nabla \chi)+(\kappa H(T-g), \chi) \Gamma_{1}=0
$$

where

$$
(T-g) \chi=\left(T-\bar{T}_{1}-\left(g-\bar{T}_{1}\right)\right) \chi \geq|\chi|^{2} \text { on } \Gamma_{1} \text {. }
$$

Thus, we obtain $|\chi|_{V_{1}}^{2}=0$ which implies $\chi=0$ and hence $T \geq \bar{T}_{1}$. Similarly, one can prove that $T \leq \bar{T}_{2}$, choosing the test function $\chi=\sup \left(T, \bar{T}_{2}\right)$.

We have also the uniqueness of solutions under the smallness assumption on $f$ and $g-T_{0}$.

Theorem 3 If $|f|_{L^{2}}$ and $\frac{1}{T_{0}}\left|g-T_{0}\right|_{L^{2}\left(\Gamma_{1}\right)}$ are sufficiently small then (6), (7) has a unique solution in $V$.

Proof: Suppose $\left(u_{i}, T_{i}-T_{0}\right) \in V, i=1,2$ are two solutions to (6), (7). Then we have

$$
\begin{aligned}
& v(\nabla \tilde{u}, \nabla \phi)+\left\langle u_{1} \cdot \nabla \tilde{u}+\tilde{u} \cdot \nabla u_{2}, \phi\right)=\gamma\left(\tilde{T} e_{d}, \phi\right) \\
& \kappa(\nabla \tilde{T}, \nabla \chi)+\left\langle u_{1} \cdot \nabla \tilde{T}+\tilde{u} \cdot \nabla\left(T_{2}-T_{0}\right), \chi\right\rangle+(\kappa H \tilde{T}, \chi)_{\Gamma_{1}}=0
\end{aligned}
$$

for $\phi \in V_{0}$ and $\chi \in V_{1}$, where $\tilde{u}=u_{1}-u_{2} \in V_{0}$ and $\tilde{T}=T_{1}-T_{2} \in V_{1}$. Setting $\phi=\tilde{u}$ and $\chi=\tilde{T}$ we obtain from (5) and (10)

$$
\begin{aligned}
& \nu|\tilde{u}|_{v_{0}}^{2} \leq M_{1}\left|u_{2}\right| v_{v_{0}}|\tilde{u}|_{v_{0}}^{2}+\gamma M_{3} M_{4}|\tilde{T}|_{v_{1}}|\tilde{u}|_{v_{0}} \\
& \kappa\left(|\nabla \tilde{T}|^{2}+H|\tilde{T}|_{\Gamma_{1}}^{2}\right) \leq M_{1}\left|T_{2}-T_{0}\right| v_{1}|\tilde{u}|_{V_{0}}|\tilde{T}|_{v_{1}} .
\end{aligned}
$$

If we set $X=|\tilde{u}|_{v_{0}}$ and $Y=|\tilde{T}|_{v_{1}}$ then this implies

$$
\left(\nu-M_{1}\left|u_{2}\right| v_{0}\right) X^{2} \leq \gamma M_{3} M_{4} X Y \text { and } \kappa Y^{2} \leq M_{1}\left|T_{2}-T_{0}\right| v_{1} X Y .
$$


Hence if $\kappa\left(\nu-M_{1}\left|u_{2}\right| v_{0}\right)-\gamma M_{1} M_{3} M_{4}\left|T_{2}-T_{0}\right| v_{1}>0$ then $X=Y=0$ and thus $\left(u_{1}, T_{1}\right)=\left(u_{2}, T_{2}\right)$. From $(11)-(12)$ we have

$$
\begin{aligned}
& \left|T_{2}-T_{0}\right|_{v_{1}} \leq H\left|g-T_{0}\right|_{L^{2}\left(\Gamma_{1}\right)} \\
& \left|u_{2}\right|_{v_{0}} \leq \frac{M_{3}}{v}\left(|f|_{L_{2}}+\gamma M_{4} H\left|g-T_{0}\right|_{L^{2}\left(\Gamma_{1}\right)}\right)
\end{aligned}
$$

Thus, if $|f|_{L^{2}}$ and $\frac{1}{T_{0}}\left|g-T_{0}\right|_{L^{2}\left(\Gamma_{1}\right)}$ are sufficiently small then (6), (7) has a unique solution.

Moreover, we can make the following demonstration of the regularity of the solution $\left(u, T-T_{0}\right) \in V$. Define the Stokes operator $A$ on $H_{0}$ by

$$
(A u, \phi)_{H_{0}}=(\nabla u, \nabla \phi) \quad \text { for } \phi \in V_{0}
$$

with domain

$$
\operatorname{dom}(A)=\left\{u \in V_{0}:|(\nabla u, \nabla \phi)| \leq c|\phi|_{H_{0}} \text { for all } \phi \in V_{0}\right\} .
$$

Then it is known [18] that $A$ is a positive self-adjoint operator on $H_{0}, \operatorname{dom}(A) \subset$ $H^{2}(\Omega)^{3}$ and $V_{0}=\operatorname{dom}\left(A^{1 / 2}\right)=\left[H_{0}, \operatorname{dom}(A)\right]_{1 / 2}$. Let $d=3$. Since

$$
|\langle u \cdot \nabla u, \phi\rangle| \leq|u|_{L^{6}}|\nabla u|_{L^{2}}|\phi|_{L^{3}}
$$

for $u, \phi \in V_{0}$ and

$$
H^{1}(\Omega) \subset L^{6}(\Omega) \text { and } \quad V_{1 / 2} \subset L^{3}(\Omega),
$$

where $V_{1 / 2}=\left[V_{0}, H_{0}\right]_{1 / 2}, u \nabla u \in V_{-1 / 2}=\operatorname{dom}\left(A^{-1 / 4}\right)$ for $u \in V_{0}$. Thus,

$u=A^{-1}\left(\rho\left(T-T_{0}\right) e_{3}+f-u \cdot \nabla u\right) \in \operatorname{dom}\left(A^{3 / 4}\right)=[V, \operatorname{dom}(A)]_{1 / 2} \subset H^{3 / 2}(\Omega)^{3}$.

Hence, $u \cdot \nabla u \in L^{2}(\Omega)^{d}$ and $u \in \operatorname{dom}(A) \subset H^{2}(\Omega)^{3}$.

\section{Necessary optimality condition}

We now show the existence of solutions to the optimal control problem (1)-(2). Let us denote by $S(g)$, the solution set of (6), (7) for $g \in L^{2}\left(\Gamma_{1}\right)$.

Theorem 4 Consider the minimization problem (1)-(2):

$$
\begin{aligned}
& \text { minimize } \quad J(g)=\varphi\left(u, T-T_{0}\right)+\frac{\beta}{2}\left|g-T_{0}\right|_{L^{2}\left(\Gamma_{1}\right)}^{2} \\
& \operatorname{over}\left(u, T-T_{0}\right) \in S(g) \text { and } g \in \mathcal{C} \text {. }
\end{aligned}
$$

Assume that $\varphi$ satisfies

$$
\begin{gathered}
\varphi(z): z=\left(u, T-T_{0}\right) \in V \rightarrow R^{+} \text {is convex and lower semicontinous } \\
\text { and } \varphi(z) \leq b_{1}|z|_{V}^{2}+b_{2} \text { for } b_{1}, b_{2} \in R^{+}
\end{gathered}
$$

Then Proble:

Proof: Let I $\beta>0, \mid g_{k}$ I $\left(u_{k}, T_{k}-T_{1}\right.$ by the same: $V \times \mathcal{C}$ since trilinear forr

for $z_{i}=\left(u_{i}\right.$ bedded into $\psi \in V$, whe: the limit $(z$, lower semici

Problem $\left(\left(u, T-T_{0}\right)\right.$ minimi

where the eq $\langle e(x)$, for $\psi=(\phi$, $a(z, \psi)=\nu$ for $z=(u, z$ denotes the 1

Theorem 5

Then there e. a for $\psi \in V a$.

Proof: It fo multiplier $\lambda^{*}$ 
Ind H. Tran

$=0$ and thus

las a unique

larity of the

$\operatorname{dom}(A) \subset$

Ius,

$H^{3 / 2}(\Omega)^{3}$.

(1)-(2). Let

ttinous

Then Problem (1)-(2) has a solution.

Proof: Let $\left(\left(u_{k}, T_{k}-T_{0}\right), g_{k}\right) \in S\left(g_{k}\right) \times \mathcal{C}$ be a minimizing sequence. Since $\beta>0 .\left|g_{k}-T_{0}\right|_{L=\left(\Gamma_{1}\right)}$ is uniformly bounded in $k$ and thus from (11)-(12) so is $\left|\left(u_{k}, T_{k}-T_{0}\right)\right|_{V}$. Hence there exists a subsequence of $\{k\}$, which will be denoted by the same index, such that $\left(u_{k}, T_{k}-T_{0}, g_{k}\right)$ converges weakly to $\left(u, T-T_{0}, g\right) \in$ $V \times C^{\prime}$ since $V \times L^{2}\left(\Gamma_{1}\right)$ is a Hilbert space and $\mathcal{C}$ is closed and convex. Define the trilinear form $\tilde{b}$ on $V^{3}$ by

$$
\left.\tilde{b}\left(z_{1}, z_{2}, \psi\right)=b\left(u_{1}, u_{2}, \phi\right)+\stackrel{5}{5} u_{1} \cdot \nabla \theta_{2}, x\right)
$$

for $z_{i}=\left(u_{i}, \theta_{i}\right), i=1,2, \psi=(\phi, x) \in V$. Since $H^{l}(\Omega)$ is compactly embedded into $L^{4}(\Omega)$ it follows from Lemma 1 that $\tilde{b}\left(z_{k}, z_{k}, \psi\right) \rightarrow \bar{b}(z, z, \psi)$ for $\psi \in V$, where $z_{k}=\left(u_{k}, T_{k}-T_{0}\right)$ and $z=\left(u, T-T_{0}\right)$. Hence, for $\psi=(\phi, \chi) \in V$ the limit $(z, g)$ satisfies $(6),(7)$ and thus $z \in S(g)$. Now, since $\varphi$ is convex and lower semicontinuous, it follows from $[5]$ that $(z, g)$ minimizes (16).

Problem (16) is equivalently written as a constrained minimization on $x=$ $\left(\left(u, T-T_{0}\right), g\right) \in X=V \times L^{2}\left(\Gamma_{1}\right)$ with

$$
\begin{gathered}
\text { minimize } J(x)=\varphi\left(u, T-T_{0}\right)+\frac{\beta}{2}\left|g-T_{0}\right|_{L^{2}\left(\Gamma_{1}\right)}^{2} \quad \text { over } x \in X \\
\text { subject to } e(x)=0 \text { and } g \in \mathcal{C}
\end{gathered}
$$

where the equality constraint $e: X \rightarrow Y=V^{*}$ is defined by (6), (7); i.e.,

$$
\langle e(x), \psi\rangle=a(z, \psi)+\tilde{b}(z, z, \psi)-\kappa\left(H\left(g-T_{0}\right), \chi\right)_{\Gamma_{1}}-(f, \phi)
$$

for $\psi=(\phi, \chi) \in V$, where

$a(z, \psi)=\nu(\nabla u, \nabla \phi)-\gamma\left(\left(T-T_{0}\right) e_{d}, \phi\right)+\kappa\left(\nabla\left(T-T_{0}\right), \nabla \chi\right)+\kappa H\left(T-T_{0}, \chi\right) \Gamma_{1}$ for $z=\left(u, T-T_{0}\right), \psi=(\phi, \chi) \in V$. Assume that $x^{*}=\left(z^{*}=\left(u^{*}, T^{*}-T_{0}\right), g^{*}\right)$ denotes the optimal pair of (16). Then we have

Theorem 5 Assume that $x^{*}$ is a regular point in the sense [14] that

$$
0 \in \operatorname{int}\left\{e^{\prime}\left(x^{*}\right)\left(v, h-g^{*}\right): v \in V \text { and } h \in \mathcal{C}\right\}
$$

Then there exists a Lagrange multiplier $\lambda^{*} \in V$ such that

$$
a\left(\lambda^{*}, \psi\right)+\tilde{b}\left(\psi, z^{*}, \lambda^{*}\right)+\tilde{b}\left(z^{*}, \psi, \lambda^{*}\right)+\left\langle\varphi^{\prime}\left(z^{*}\right), \psi\right\rangle=0
$$

for $\psi \in V$ and

$$
\left(\beta g^{*}-\kappa H \lambda_{2}^{*}, h-g^{*}\right)_{\Gamma_{1}} \geq 0 \quad \text { for all } h \in \mathcal{C} .
$$

Proof: It follows from [14] that if (19) is satisfied, then there exists a Lagrange multiplier $\lambda^{*}=\left(\lambda_{1}^{*}, \lambda_{2}^{*}\right) \in V$ such that

$$
\left\langle\varphi^{\prime}\left(z^{*}\right), \psi\right)+\beta\left(g^{*}, h-g^{*}\right) \Gamma_{1}+e^{\prime}\left(x^{*}\right)\left(\psi, h-g^{*}\right) \geq 0
$$

\section{ORIGIUAL PAGE IS OF. POOR QUALITY}


for all $\psi \in V$ and $h \in \mathcal{C}$, that is

$$
\begin{aligned}
& \left\langle\varphi^{\prime}\left(z^{*}\right), \psi\right\rangle+\beta\left(g^{*}, h-g^{*}\right) \Gamma_{1}+a\left(\lambda^{*}, \psi\right) \\
& \quad+\tilde{b}\left(\psi, z^{*}, \lambda^{*}\right)+\tilde{b}\left(z^{*}, \psi, \lambda^{*}\right)-\kappa H\left(h-g^{*}, \lambda_{2}^{*}\right)_{\Gamma_{1}} \geq 0
\end{aligned}
$$

for all $\psi \in V$ and $h \in K$. Setting $\psi=0$, we obtain (21). Next, setting $h=g^{*}$ in (22), we obtain (20).

Concerning the regular point condition (19), we have

Lemma 6 If $g^{*} \in \operatorname{int}(\mathcal{C})$ then the regular point condition (19) is equivalent to the condition that for $v=\left(v_{1}, v_{2}\right) \in V$

$$
\begin{aligned}
& a(v, \psi)+\tilde{b}\left(\psi, z^{*}, v\right)+\tilde{b}\left(z^{*}, \psi, v\right)=0 \\
& \text { for all } \psi \in V \text { and } v_{2}=0 \text { on } \Gamma_{1}
\end{aligned}
$$

implies $v=0$.

Proof: If $g^{*} \in \operatorname{int}(\mathcal{C})$ then (19) is equivalent to the condition that $G=e^{\prime}\left(x^{*}\right)$ is surjective. Define the linear map $C \in \mathcal{L}(X, V)$ by $C(v, h)=\xi$ where $\xi \in V$ is the unique solution to

$$
a(\xi, \psi)+\tilde{b}\left(v, z^{*}, \psi\right)+\tilde{b}\left(z^{*}, v, \psi\right)-\kappa H(h, \chi)_{r_{1}}=0 \quad \text { for } \psi=(\phi, \chi) \in V
$$

Then, since $H^{1}(\Omega)$ is embedded compactly to $L^{4}(\Omega)$, Lemma 1 implies that $C$ is compact. Thus, by the Banach closed range theorem and the Riesz-Schauder theorem, $e^{\prime}\left(x^{*}\right)(v, h)$ is surjective if and only if $\operatorname{ker}\left(G^{*}\right)=\{0\}$ [4], which is equivalent to $(21)$.

\section{Augmented Lagrangian method}

In this section we discuss applications of the augmented Lagrangian method for the constrained minimization problem (17). The augmented Lagrangian method [8], [15] is based on an equivalent formulation of (17):

$$
\text { minimize } J(x)+\frac{c}{2}|e(x)|_{Y}^{2} \quad \text { over } x \in X \text { and } g \in \mathcal{C} .
$$

subject to $e(x)=0$, where $x=\left(\left(u, T-T_{0}\right), g\right) \in X=V \times L^{2}\left(\Gamma_{1}\right)$ and $c>$ 0 is the penalty parameter. The augmented Lagrangian algorithm [8], [15] is the multiplier method applied to (17), i.e., it involves a sequence of minimizations of the functional

$$
\begin{gathered}
L_{c_{k}}\left(x ; \lambda^{k}\right)=J(x)+\left\langle\lambda^{k}, e(x)\right\rangle+\frac{c}{2}|e(x)|_{Y}^{2} \\
\text { subject to } g \in \mathcal{C},
\end{gathered}
$$


where the multiplier sequence $\left\{\lambda^{k}\right\}$ in $Y^{*}$ is generated by the first order update

$$
\lambda^{k+1}=\lambda^{k}+c_{k} e\left(x_{k}\right) \text {. }
$$

for $k \geq 1$, where $x_{k}$ is a minimizer of $L_{c_{k}}\left(\cdot, \lambda^{k}\right)$ and we assumed that $Y^{*}=Y$. otherwise each element in $Y^{*}$ has its Riesz representation. To carry out this iteration, a sequence of monotonically nondecreasing. positive real numbers $\left\{c_{k}\right\}$. $c_{1}>c_{0} \geq 0$ and a start up value $\lambda^{1}$ for the Lagrange multiplier for the equality constraint $e(x)=0$ need to be chosen. The convergence fesults of the augmented

to the Lagrangian method for the infinite dimensional optimization problem are established, for example, in [9], [16]. The augmented Lagrangian method is a hybrid of the penalty method (i.e., $\lambda^{k}=0$ ) and the Lagrange muitiplier method (i.e.. $c_{k}=0$ ) and combines good properties of both. It overcomes the difficulty of the penalty method which requires a large value of $c_{k}$. The augmented functional $L_{c_{k}}\left(x, \lambda^{k}\right)$ is locally strictly convex provided that $\lambda^{k}$ is sufficiently close to $\lambda^{*}$ and the second order optimality condition

$$
\begin{aligned}
& L_{0}^{\prime \prime}\left(x^{*}, \lambda^{*}\right)((v, h),(v, h)) \geq \sigma\left(|v|_{V}^{2}+|h|_{u}^{2}\right) \\
& \quad \text { for all }(v, h) \in X \text { satisfying } e^{\prime}\left(x^{*}\right)(v . h)=0 .
\end{aligned}
$$

for some $\sigma>0$, is satisfied. Here, $L_{0}^{\prime \prime}\left(x^{*}, \lambda^{*}\right)$ denotes the bilinear form that characterizes the second derivative of $L_{0}(x, \lambda)=J(x)+\{\lambda, e(x)\rangle$ with respect to $x$ at $x^{*}$. That is, it is not necessary that the cost functional $J$ be (locally) convex, which is required for convergence of the multiplier method. The algorithm (25)-(26) has been successfully applied to parameter estimation problems in elliptic PDEs [10], [12] and optimal control problems for 2-D incompressible Navier-Stokes [4]. The first order update (26) provides Q-linear convergence of the iterates $x_{k}$ in $X$. In [11] we have investigated a second order update scheme for the augmented Lagrangian method. In what follows we assume that $g^{*} \in$ int $\left(C^{\prime}\right)$. Thus. (19) reduces to $e^{\prime}\left(x^{*}\right)$ is surjective (see Lemma 6 ). Hence the necessary condition implies that

$$
L_{c}^{\prime}\left(x^{*}, \lambda^{*}\right)=0 \text { and. } e\left(x^{*}\right)=0 \text {, }
$$

for all $c \geq 0$. An algorithm proposed in [11] applies Newton's method to (28). The resulting algorithm is stated as: given a current iterate $(x, \lambda)$ the next iterate $\left(x_{+}, \lambda_{+}\right)$satisfies

$$
\left(\begin{array}{cc}
L_{c}^{\prime \prime}(x, \lambda) & e^{\prime}(x)^{*} \\
e^{\prime}(x) & 0
\end{array}\right)\left(\begin{array}{c}
x_{+}-x \\
\lambda_{+}-\lambda
\end{array}\right)=-\left(\begin{array}{c}
L_{c}^{\prime}(x, \lambda) \\
e(x)
\end{array}\right) .
$$

Note that

$$
L_{c}^{\prime}(x, \lambda)=L_{0}^{\prime}(x, \lambda+c e(x))
$$

and

$$
L_{c}^{\prime \prime}(x, \lambda)=L_{0}^{\prime \prime}(x, \lambda+c e(x))+c\left\langle e^{\prime}(x)(\cdot), e^{\prime}(x)(\cdot)\right\rangle
$$

\section{ORIGINAL PAGE IS OF, POOR QUALITY}


Consequently, suppose $\left|(x, \lambda)-\left(x^{*}, \lambda^{*}\right)\right|$ is sufficiently small. Then it follows from (27) [11] that $L_{c}^{\prime \prime}(x, \lambda)$ is coercive on $X \times Y$. Thus equation (29) can be regarded as a general Stokes equation. Following an argument due to Bertsekes [2], we can avoid forming $L_{c}^{\prime \prime}$ during the iteration. From the second equation of (29) we have $e^{\prime}(x)\left(x_{+}-x\right)=-e(x)$. Thus the first equation can be written as

$$
L_{0}^{\prime \prime}(x, \lambda+c e(x))\left(x_{+}-x\right)+e^{\prime}(x)^{*}\left(\lambda_{+}-(\lambda+c e(x))\right)=-L_{0}^{\prime}(x, \lambda+c e(x))
$$

and hence (29) is equivalent to

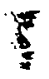

$$
\left(\begin{array}{cc}
L_{0}^{\prime \prime}(x, \hat{\lambda}) & e^{\prime}(x)^{*} \\
e^{\prime}(x) & 0
\end{array}\right)\left(\begin{array}{c}
x_{+}-x \\
\lambda_{+}-\hat{\lambda}
\end{array}\right)=-\left(\begin{array}{c}
L_{0}^{\prime}(x, \hat{\lambda}) \\
e(x)
\end{array}\right)
$$

$$
\text { where } \hat{\lambda}=\lambda+c e(x) \text {. }
$$

Note that $\hat{\lambda}$ is nothing but the first order update of the Lagrange multiplier if the current iterate $x$ minimizes $L_{c}(x, \lambda)$. Equation (31) is more advantageous than (29) since the squaring term $c e^{\prime}(x)^{*} e^{\prime}(x)$ is absorbed and less calculation is involved. If we define a matrix operator $S$ on $X \times Y$ by

$$
S(x, \lambda)=\left(\begin{array}{cc}
L_{0}^{\prime \prime}(x, \lambda) & e^{\prime}(x)^{*} \\
e^{\prime}(x) & 0
\end{array}\right),
$$

then it follows from (27) that $S\left(x^{*}, \lambda^{*}\right)$ is boundedly invertible. Thus, if $(x, \lambda)$ is sufficiently close to $\left(x^{*}, \lambda^{*}\right)$, then equation (31) has a unique solution. We summarize our discussions as

\section{Algorithm 1}

(1) Choose $\lambda^{1} \in Y, c>\bar{c} \geq 0$, and set $\hat{c}=c-\bar{c}, k=1$.

(2) Determine $x=\left(\left(u, T-T_{0}\right), g\right) \in V \times \mathcal{C}$ such that

$$
L_{c}\left(x, \lambda^{k}\right) \leq L_{c}\left(x^{*}, \lambda^{k}\right)=f\left(x^{*}\right) .
$$

(3) Set $\hat{\lambda}=\lambda^{k}+\hat{c} e(x)$.

(4) Solve for $\left(x_{+}, \lambda_{+}\right) \in X \times Y$ :

$$
S(x, \hat{\lambda})\left(\begin{array}{c}
x_{+}-x \\
\lambda_{+}-\hat{\lambda}
\end{array}\right)=-\left(\begin{array}{c}
L_{0}^{\prime}(x, \hat{\lambda}) \\
e(x)
\end{array}\right) .
$$

(5) Set $x_{k+1}=x_{+}$and $\lambda^{k+1}=\lambda_{+}$. If the convergence criterion is not satisfied then set $k=k+1$ and go to (2).

Remark. A duced to the reduction o ample, if $x$ Assume tha ciently sma Q-quadratic

\section{Hybrio}

In this sectic retical result dimensional

minimiz

subject to (2 interior of $\Gamma$

$$
\begin{aligned}
& T(0, x: \\
& T\left(x_{1}, 1\right.
\end{aligned}
$$

with a refere $\left(x_{1}, 0\right), \frac{1.5}{5} i$ (32) is chost flow with $L$ for the flow indicates tha of physical $\mathrm{F}$

The prot has dimensic given by $v=$ $5.5 \times 10^{-5}$ : and $u^{1}$, respi $u^{1}$ does and (24) is formu transport of $f$ reference ten 
slows de re. es $[2]$, ir (29)

e cur-

slved.

$\lambda)$ is

sum-

then

Remark. A variant of Algorithm 1 is obtained by skipping step (2). Then it is reduced to the Newton method applied to equation (29). Step (2) implies a suffic:ent reduction of the merit functional (the augmented Lagrange functional). For example, if $x=(z, g)$ minimizes $L_{c}\left(\cdot, \lambda^{k}\right)$ over $V \times C$ then step (2) is completed. Assume that (19) and (27) hold. It is proved in [11] that if $\left|\lambda^{\prime}-\lambda^{*}\right|_{\gamma}$ is surficiently small. then Algorithm 1 is well-posed and $\left(x^{*}, \lambda^{k}\right)$ converges to $\left(x^{*}, \lambda^{*}\right)$ Q-quadratically.

\section{Hybrid method and test example}

In this section we present an example to demonstrate the applicability of our theoretical results and proposed algorithm. We consider the optimal control of the two dimensional stationary thermally driven cavity flow

$$
\begin{gathered}
\operatorname{minimize} \quad J(g)=\frac{1}{2} \int_{\Omega}\left|u-u_{d}\right|^{2}+\left|T-T_{d}\right|^{2} d x+\frac{\beta}{2}\left|g-T_{0}\right|_{L^{2}\left(\Gamma_{1}\right)}^{2} \\
\text { over } g \in L^{2}\left(\Gamma_{1}\right)
\end{gathered}
$$

subject to (2), where $\Omega=(0, L)^{2}, \Gamma_{1}=\left(x_{1}, 1\right), 0<x_{1}<L$ and $\Gamma_{0}$ is the relative interior of $\Gamma-\Gamma_{1}$. On $\Gamma_{0}$ we have the Dirichlet boundary condition:

$$
\begin{aligned}
& T\left(0, x_{2}\right)=T\left(L, x_{2}\right)=T_{0}, \quad 0<x_{2}<L \\
& T\left(x_{1}, 0\right)=T_{0}-50 \min \left(1, \frac{2}{3}\left(2.5-\left|\frac{5}{L} x_{1}-2.5\right|\right), \quad 0<x_{1}<L .\right.
\end{aligned}
$$

with a reference temperature $T_{0}=1350^{\circ} \mathrm{K}$, and the temperature on the substrate $\left(x_{1}, 0\right), \frac{1.5}{5} L \leq x_{1} \leq \frac{3.5}{5} L$ is $1300^{\circ} K$. The desired state $\left(u_{d}, T_{d}\right)$ appearing in (32) is chosen as follows. $u_{d}=L u^{0}$ where $\left(u^{0}, T^{0}\right)$ is the solution pair for the flow with $L=1$ and $g=T_{0}$ and $T_{d}=T^{1}$ where $\left(u^{1}, T^{1}\right)$ is the solution pair for the flow with $L=5$ and $g=T_{0}$. Here, our numerical calculation strongly indicates that the solution to (2) is unique and (dynamically) stable for the range of physical parameters that we chosen for our calculation.

The problem is scaled so that the velocity field $u$ has dimension $\mathrm{cm} / \mathrm{sec}$ and $T$ has dimension $K^{\circ}$. The constants $\nu, \kappa$ are chosen for $P_{2}$ at $3 a t m$ pressure and are given by $\nu=.155$ and $\kappa=.110 . H, \beta$ and $L$ are set to be $H=100, \beta=\frac{1000}{1350^{2}}=$ $5.5 \times 10^{-5}$ and $L=5$ respectively. Figures 1 and 2 show the vector field of $u^{0}$ and $u^{1}$, respectively. It can be observed that $u^{0}$ has more vertical transport than $u^{i}$ does and $u^{1}$ is confined in the two bottom corners. Hence the cost-functional (24) is formulated so that the thermal control $g$ on the top $\Gamma_{1}$ increases the vertical transport of flow with $L=5$ while retaining the temperature distribution $T^{1}$ at the reference temperature $g=T_{0}$.

\section{ORIGINAL PAGE IS OF POOR QUALTY}




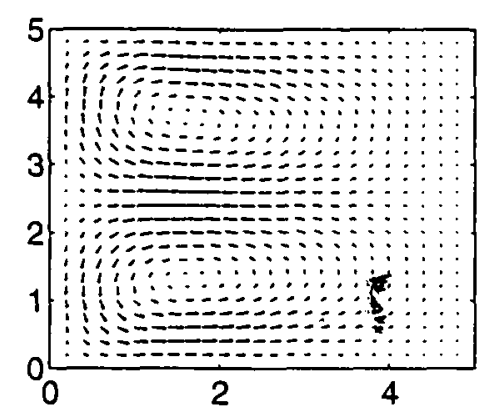

Figure 2. The uncontrolled flow.

The second order augmented Lagrangian method (Algorithm 1) described in Section 4 is used to solve problem (32). To obtain a good starting value $\lambda^{1}$ for the Lagrange multiplier in Algorithm 1, we employed a few steps of the gradient method. The gradient of the cost functional can be calculated by the adjoint equation as follows. Consider the cost functional $J(z, g)$ subject to $E(z, g)=0$ where $z \in V$ and $g \in U$. Assume that $V$ and $U$ are Hilbert spaces, $J(z, g): X=$ $V \times U \rightarrow R$ is Fréchet differentiable and $E(z, g): X \rightarrow Y$ is continuously Fréchet differentiable in a neighborhood of $x_{0}=\left(z_{0}, g_{0}\right)$. Suppose that $E_{z}\left(x_{0}\right) \in \mathcal{L}(V, Y)$, the $F$-derivative of $E$ with respect to $z$ at $x_{0}$, has a bounded inverse. Then by the implicit function theorem, there exists a unique $C^{1}$ mapping $\Psi$ defined in a neighborhood $N$ of $g_{0}$ in $U, \Psi: U \rightarrow V$ such that $\Psi\left(g_{0}\right)=z_{0}$ and $E(\Psi(g), g)=0$ for $g \in N$. Then the $F$-derivative $d$ of $J(\Psi(g), g)$ with respect to $g$ in $N$ exists and is given by

$$
d=J_{g}+E_{g}^{*} \psi
$$


where $\psi \equiv Y$ satisties the adjoint equation

$$
E_{:}^{*} \psi+J_{z}=0
$$

Here we assume that $V, U$ and $Y$ are identined with their dual spaces. In fact. if $v=\Psi^{\prime}(h), h \equiv U$ then

$$
(d . h)_{U}=\left(J_{g}, h\right)_{U}+\left(J_{z}, v\right)_{V} .
$$

and $v \in V$ satisfies

$$
E_{z} v+E_{g} h=0
$$

Hence, for $h \in U$

$$
(d, h)_{U}=\left(J_{g}, h\right)_{U}-\left(E_{z}^{*} \psi, v\right)_{V}=\left(J_{3}, h\right)_{U}+\left(\lambda, E_{z} h\right)_{V}=\left(J_{s}+E_{z}^{*} \psi, h\right)
$$

which implies (34). The projected gradient method can be written as:

\section{Algorithm 2}

(1) Choose $g_{1} \in U$ and set $k=1$.

(2) Let $z_{k}$ be a solution of $E\left(z, g_{k}\right)=0, \psi_{k}$ be the solution of $E_{z}\left(z_{k}, g_{k}\right)^{*} \psi_{k}+$ $J_{z}\left(z_{k}, g_{k}\right)=0$ and set $d_{k}=J_{g}\left(z_{k}, g_{k}\right)+E_{g}\left(z_{k}, g_{k}\right)^{*} \psi_{k}$.

(3) Set $d_{k}=J_{g}\left(z_{k}, g_{k}\right)$ and determine $\alpha_{k} \geq 0$ such that $J\left(\Psi\left(g_{\alpha}\right), g_{\alpha}\right)$ is minimized where $g_{\alpha}=\operatorname{Projc}\left(g_{k}-\alpha d_{k}\right)$.

(4) Set $g_{k+1}=g_{k}-\alpha_{k} d_{k}$. If the convergence criterion is not satisfied, then set $k=k+1$ and go to Step (2).

In our specific example equation (35) is written as

$$
\begin{gathered}
-\nu \Delta \lambda-u \cdot \nabla \lambda+\lambda^{\prime} \nabla u-\left(T-T_{0}\right) \nabla \mu+\nabla q+u-u_{d}=0 \\
\nabla \cdot \lambda=0 \text { and } \lambda \mid r=0 \\
-\kappa \Delta \mu-u \cdot \nabla \mu-\frac{g}{T_{0}} \lambda_{2}+T-T_{d}=0 \\
\mu=0 \text { on } \Gamma_{0} \text { and } n \cdot \nabla \mu+\kappa H \mu=0 \text { on } \Gamma_{1},
\end{gathered}
$$

where $\psi=(\lambda, \mu) \in V=V_{0} \times V_{1}, q \in L^{2}(\Omega)$ and

$$
E_{:}^{*} \psi=-\kappa H \mu \quad \text { on } \Gamma_{1} \text {. }
$$

We used the mixed-finite element method [7] based on the Legendre polynomiais to approximate problem (1)-(2) numerically. Detailed discussions about the method are given in [13]. In our implementation we calculated the adjoint system for the approximated problem and solved equations (2) and (36) using GMVES [3]. The specinic implementation of GMRES applied to our example is described in [13] in detail. Concerning the divergence-free constraint $\nabla \cdot u=0$, we employed

\section{ORIGHNAL PAGE IS OF POOR QUALITY}


the feasible method, projecting the first equation onto the divergence-free space $V_{0}$ as in [4], [6], [13]. The line search in Step 3 of Algorithm 2 was performed by the linearization of the constraint $E(z, g)=0$ at $\left(z_{k}, g_{k}\right)$ since the cost functional is quadratic. That is, if $v_{k}$ is the solution to $E_{z}\left(z_{k}, g_{k}\right) v_{k}+E_{g}\left(z_{k}, g_{k}\right) d_{k}=0$ then $\alpha>0$ is chosen so that $J\left(z_{k}-\alpha v_{k}, g_{k}-\alpha d_{k}\right)$ is minimized.

For this specific example, three steps of Algorithm 2 were performed. We then set $x^{1}$ and $\lambda^{1}$ as $x^{1}=\left(z_{4}, g_{4}\right)$ and $\lambda^{1}=\psi_{3}$ for Algorithm 1 without Step 2. The matrix operator $S(x, \lambda)$ was calculated for the approximated system and the resulting linear equation ( 31 ) was again solved by GMRES.

The calculations were performed using a $20 \times 20$ Cartesian product of Legendre polynomials, choosing $c=1$ and $g_{1}=0$. Algorithm 1 was terminated after three iterates, since the necessary and sufficient optimality condition (28) was satisfied within a residual norm of $1 \times 10^{-7}$.

We may compare this rapid convergence of the hybrid method with the results of using either algorithm by itself. Algorithm 2 did not fully converge after 50 iterates. Algorithm 1, with the start-up $x^{1}=\left(\left(u^{1}, T^{1}\right), 0\right) \in X$ and $\lambda^{1}=0$, also failed to converge. Thus, the use of the hybrid method combining the gradient method and the second-order augmented Lagrangian was essential for the success of our numerical calculations. Figure 3 shows the iterates $g_{k}$ (the first three curves from top to middle) for Algorithm 1 and the (calculated) optimal control $g^{*}$ (the lowest curve). The iterates for Algorithm 1 are not shown because they coincide with $g^{*}$ within the accuracy of the plotting. Figure 4 shows the resulting vector field $u^{*}$ which corresponds to $g^{*}$. It shows clearly that the vertical transport of flow is increased.

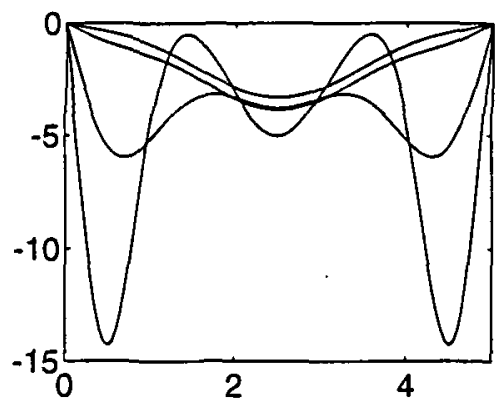

Figure 3. Optimal control iterates.

\section{References}

[1] F. Abergel : Theor. Com;

[2] D. Bertseka Academic, I

[3] P. Brown an tions, SIAM

[4] M. Desai as Cont., to ap:

[5] I. Ekeland a Holland, Ar

[6] R. Glowins Springer, B

[7] V. Girault ar tions, Sprin

[8] M.R.Hester 1968, 303-

[9] K. Ito and I inequality $\mathrm{c}$

[10] K. Ito and $\mathrm{F}$ timation in

[11] K. Ito and I der update : appear. 


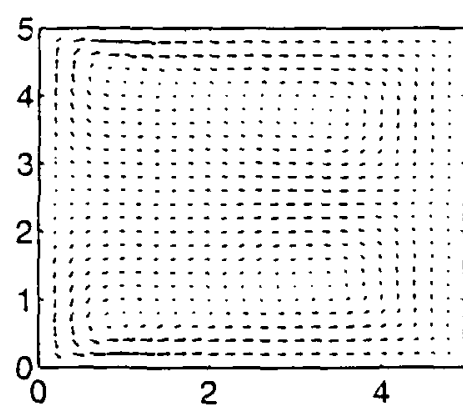

Figure 4. The controlled How.

\section{References}

[1] F. Abergel and R. Temam: On some control problems in Huid mechanics. Theor. Comp. Fluid Mech. 1, 1990, 303-325.

[2] D. Bertsekas; Constraint Optimization an Lagrange Multiplier Methods. Academic, Paris, 1982.

[3] P. Brown and Y. Saad; Hybrid Krylov methods for nonlinear systems of equations, SIAM J. Sci. Statist. Comput. 11, 1990, 450-481.

[4] M. Desai and K. Ito; Optimal control of Navier-Stokes equations, SIAM J. Cont., to appear.

[5] I. Ekeland and R. Temam; Convex Analysis and Variational Problems, North Holland, Amsterdam, 1976.

[6] R. Glowinski; Numerical Methods for Nonlinear Variational Problems, Springer, Berlin, 1984.

[7] V. Girault and P.-A. Raviart; Finite Element Methods for Navier-Stokes Equations, Springer, Berlin, 1984.

[8] M.R.Hestenes; Multipiier and gradient methods, J. Opt. Theory Appl. 4, 1968, 303-320.

[9] K. Ito and K. Kunisch; The augmented Lagrangian method tor equality and inequality constraints in Hilbert spaces, Math. Prog. 46 ,1990. 341-360.

[10] K. Ito and K. Kunisch; The augmented Lagrangian method for parameter estimation in elliptic systems, SIAM J. Cont. \& Optm. 28, 1990, 113-136.

[11] K. Ito and K. Kunisch; The augmented Lagrangian method with second order update and its application to parameter estimation in elliptic systems, to appear.

\section{ORIGINAL PAGE IS OF POOR QUALTTY}


[12] K. Ito, M. Kroller, and K. Kunisch; A numerical study of an augmented Lagrangian method for the estimation of parameters in elliptic systems, SIAM J. Sci. Stat. Comput. 12, 1991, 884-910.

[13] K. Ito, J. Scroggs. and H. Tran; Mathematical issues in optimal design of a vapor transport reactor, Flon: Controi (Ed. by M. Gunzburger), Springer, 1995, to appear.

[14] H. Maurer and J. Zowe; First and second-order necessary and sufficient optimality conditions for infinite-dimensional programming problems, Math. Prog. 16, 1979, 98-110.

[15] M. Powell: A method for nonlinear constraints in minimization problems, in Optimization (Ed. by R. Fletcher), Academic, New York, 1969.

[16] V. Polyak and Tret yakov; The method of penalty estimates for conditional extremum problems. Z. Vychisl. Mat. i Mat. Fiz. 13 1973, 34-46.

[17] R. Temam; Navier-Stokes Equations and Nonlinear Functional Analysis. SIAM, Philadelphia, 1983.

[18] R. Temam; Infinite Dimensional Dynamical Systems in Mechanics and Physics, Springer. New York, 1988.

[19] G. Troianiello; Elliptic Differential Equations and Obstacle Problems, Plenum, New' York. 1987. 